\title{
A generic view on the theorems of Brouwer and Schauder
}

\author{
Tudor Zamfirescu \\ Fachbereich Mathematik, Universität Dortmund, W-4600 Dortmund, Germany \\ Received 28 June 1991; in final form 22 July 1992
}

Several generic fixed point theorems have been established by De Blasi $[5,6]$, Myjak [9], De Blasi and Myjak [7], Myjak and Sampalmieri [10], Butler [4], Dominguez Benavides [8], Vidossich [12], to quote just a few. However it seems that the well-known and important theorem of Schauder [11] and its finitedimensional version, Brouwer's theorem [3], have not yet been generically investigated. We now fill this gap.

For a survey of generic results on convex bodies in Euclidean spaces, see [15].

\section{A large set of fixed points}

Let $E$ be a Banach space and consider a compact convex set $K \subset E$ with more than one point. By the Schauder theorem, every continuous function $f: K \rightarrow K$ has at least one fixed point. We shall show here that, in the sense of Baire categories, most continuous functions have uncountably many fixed points. More precisely, the set of all their fixed points is homeomorphic to the Cantor set. This sharply contrasts with the generic finiteness of the fixed point set found by De Blasi [6] in another space of functions and with the behaviour of the non-expansive mappings which have, generically, a single fixed point $([12]$, see also $[9$, p. 29]). Of course, the space $\mathscr{C}(K)$ of all continuous functions $f: K \rightarrow K$, equipped with the metric of uniform convergence, is complete, hence a Baire space. "Most" means "all, except those in a first category set" (so, a property is generic if it is shared by most elements).

For $f \in \mathscr{C}(K)$, let $F_{f}$ be the set of all fixed points of $f$. For $x \in E, B(x, r)$ denotes the open ball of centre $x$ and radius $r$.

The proof of Theorem A below will make use of the following recent result.

Lemma. Let $C$ be a convex closed set in $E, f: C \rightarrow C$ be a map with $\overline{f(C)}$ compact and $\varepsilon>0$ be given. Then there exists a map $g: C \rightarrow C$ such that $F_{g}$ is finite and $\|f-g\|<\varepsilon$.

This result was attributed in [2] to Brown and extended to finite unions of convex sets by Baillon and Rallis [2].

Theorem A. For most functions in $\mathscr{C}(K)$ the set of fixed points is homeomorphic to the Cantor set. 
Proof. Clearly, $F_{f}$ is closed for any $f \in \mathscr{C}(K)$. Thus we only have to show that, for most $f \in \mathscr{C}(K), F_{f}$ has no isolated point and is totally disconnected. We first prove that $F_{f}$ has no isolated point. Let $\mathscr{C}_{n}$ be the set of all functions $f \in \mathscr{C}(K)$ admitting a fixed point $y \in K$ such that

$$
F_{f} \cap B\left(y, n^{-1}\right)=\{y\} \quad(n \in \mathbb{N}) .
$$

Clearly, $\bigcup_{n} \mathscr{C}_{n}$ is precisely the set of all $f \in \mathscr{C}(K)$ admitting isolated fixed points, so it suffices to prove that $\mathscr{C}_{n}$ is nowhere dense, for arbitrary $n$.

Let $\mathscr{O} \subset \mathscr{C}(K)$ be open and, if $\mathscr{O} \cap \mathscr{C}_{n} \neq \emptyset$, take $f \in \mathcal{O} \cap \mathscr{C}_{n}$. We shall find an open set in $\mathscr{O} \backslash \mathscr{C}_{n}$.

From the covering $\left\{B\left(y,(2 n)^{-1}\right): y \in F_{f}\right\}$ of $F_{f}$ we select a finite subcovering $\left\{B\left(y,(2 n)^{-1}\right): y \in Y\right\}$. Clearly, for some $v \in\left(0,(2 n)^{-1}\right)$, if $y$ and $y^{\prime}$ are distinct points of $Y$, then

$$
B(y, v) \cap B\left(y^{\prime}, v\right)=\varnothing .
$$

Let $\varepsilon \in(0, v)$ be such that $\|f-g\|<\varepsilon$ implies $g \in \mathcal{O}$. The set $K$ being compact, we can find a number $\delta \in(0, \varepsilon / 6)$ such that $x, x^{\prime} \in K$ and $\left\|x-x^{\prime}\right\|<4 \delta$ imply $\left\|f(x)-f\left(x^{\prime}\right)\right\|<\varepsilon / 3$.

Since $L=K \backslash \bigcup_{y \in Y} B\left(y,(2 n)^{-1}\right)$ is compact and disjoint from $F_{f}$ if it is nonempty, for some $\omega>0$ the inequality $\|f(x)-x\| \geqq \omega$ holds for any $x \in L$.

For every point $y \in Y$, consider a point $z_{y} \in K$ and a number $\alpha$ such that $0<\alpha<\omega$, if $L \neq \phi$,

$$
B\left(z_{y}, 2 \alpha\right) \subset B(y, \delta) \text { and } B(y, 2 \alpha) \cap B\left(z_{y}, 2 \alpha\right)=\varnothing .
$$

We define a function $f^{*}: K \rightarrow K$ as follows:

$$
f^{*}(x)= \begin{cases}f(x) & \text { if } x \notin \bigcup_{y \in Y} B(y, 4 \delta), \\ f(y+(2 \lambda-4 \delta) v) & \text { if } x=y+\lambda v \text { and } 2 \delta \leqq \lambda<4 \delta, \\ y+(2 \delta-\lambda) v & \text { if } x=y+\lambda v \text { and } \delta \leqq \lambda<2 \delta, \\ x & \text { if } x=y+\lambda v, \quad 2 \alpha \leqq \lambda<\delta \\ y+(2 \lambda-2 \alpha) v & \text { if } x=y+\lambda v \text { and } \alpha \leqq \lambda<2 \alpha, \\ y & \text { if } x=y+\lambda v \text { and } 0 \leqq \lambda<\alpha, \\ z_{y}+(2 \lambda-2 \alpha) v & \text { if } x=z_{y}+\lambda v \text { and } \alpha \leqq \lambda<2 \alpha, \\ z_{y} & \text { if } x=z_{y}+\lambda v \text { and } 0 \leqq \lambda<\alpha,\end{cases}
$$

where, each time, $y$ is some point in $Y$ and $v$ is some unit vector. It is easily checked that $f^{*}$ is continuous and $\left\|f^{*}-f\right\|<2 \varepsilon / 3$.

Thus, for every $y \in Y, f^{*}(\overline{B(y, \alpha)} \cap K)=\{y\}$ and $f^{*}\left(\overline{B\left(z_{y}, \alpha\right)} \cap K\right)=\left\{z_{y}\right\}$. Therefore, for every function $g \in \mathscr{C}(K)$ with $\left\|f^{*}-g\right\|<\alpha$,

$$
g(\overline{B(y, \alpha)} \cap K) \subset \overline{B(y, \alpha)} \cap K
$$

and

$$
g\left(\overline{B\left(z_{y}, \alpha\right)} \cap K\right) \subset \overline{B\left(z_{y}, \alpha\right)} \cap K
$$


By Schauder's theorem, $g$ has a fixed point in each of the sets $\overline{B(y, \alpha)}$ and $\overline{B\left(z_{y}, \alpha\right)}$, where $y \in Y$.

We investigate now the fixed points of $g$. Since $\alpha<\omega, g$ has no fixed points in $L$. To any possibly existing fixed point in $K \backslash L$ there is a point $y \in Y$ at distance less than $(2 n)^{-1}$ and a pair of fixed points, the one in $\overline{B(y, \alpha)}$ and the other in $\overline{B\left(z_{y}, \alpha\right)}$. These three fixed points of $g$ have pairwise distances less than $n^{-1}$, and the last two are certainly distinct. Therefore $g \notin \mathscr{C}_{n}$. On the other hand $g \in \mathcal{O}$ because $\|f-g\|<\varepsilon$. Hence, for most $f \in \mathscr{C}(K), F_{f}$ has no isolated point.

Now we show that $F_{f}$ is totally disconnected for most $f \in \mathscr{C}(K)$. Let $\mathscr{C}_{n}^{\prime}$ be the set of all functions $f \in \mathscr{C}(K)$ such that $F_{f}$ has a component of diameter at least $n^{-1}$. It suffices to prove that $\mathscr{C}_{n}^{\prime}$ is nowhere dense.

Let $\mathcal{O} \subset \mathscr{C}(K)$ be open. By the lemma, there is some $f \in \mathcal{O}$ with finite $F_{f}$. Let $\alpha$ be the minimum distance between distinct points in $F_{f}$, or equal to 1 if $F_{f}$ consists of a single point. Let $\beta=\min \left\{\alpha / 3,(3 n)^{-1}\right\}$. The set

$$
L^{\prime}=K \backslash \bigcup_{x \in F_{f}} B(x, \beta)
$$

being compact and disjoint from $F_{f}$ (or empty), for some $\omega>0$ the inequality $\|f(x)-x\| \geqq \omega$ holds for all $x \in L^{\prime}$.

For every function $g \in \mathscr{C}(K)$ with $\|f-g\|<\omega$ we have $F_{g} \cap L^{\prime}=\varnothing$ because $\|g(x)-x\| \geqq\|f(x)-x\|-\|f(x)-g(x)\|>0$ for all $x \in L^{\prime}$. So each component of $F_{g}$ lies in some open ball $B(x, \beta)$ with $x \in F_{f}$ and therefore has a diameter less than $n^{-1}$. This shows that $\mathscr{C}_{n}^{\prime}$ is nowhere dense and the proof is finished.

\section{A small set of fixed points}

Let now $K \subset \mathbb{R}^{d}$ be compact, convex and with nonempty interior. By the wellknown Brouwer fixed point theorem [3], for any $f \in \mathscr{C}(K)$, the set $F_{f}$ of all fixed points of $f$ is nonempty. By Theorem A, for most $f \in \mathscr{C}(K), F_{f}$ is uncountable. But, otherwise, how large is $F_{f}$ for most $f$ ? In particular, is it of positive measure? We answer here this question.

As a matter of notation, for any sets $A, B \subset \mathbb{R}^{d}$ and point $x \in \mathbb{R}^{d}$,

$$
\Delta(x, A)=\inf _{y \in A}\|x-y\| \quad \text { and } \quad D(A, B)=\inf _{x \in A} \Delta(x, B) .
$$

Let $M \subset \mathbb{R}^{d}$ and $x \in M$. For any $\varepsilon>0$, let $\gamma_{\varepsilon}(x)$ be the radius of the largest open ball with centre in $\overline{B(x, \varepsilon)}$ and disjoint from $M$. Then

$$
\rho_{M}=\inf _{x \in M} \lim \sup _{\varepsilon \rightarrow 0} \gamma_{\varepsilon}(x) / \varepsilon
$$

is called the porosity of $M$. The set $M$ is called porous if $\rho_{M}>0$ and strongly porous if $\rho_{M}=1$.

Let again $M \subset \mathbb{R}^{d}$ and $x \in M$. For any $\varepsilon>0$, let $\delta_{\varepsilon}(x)$ be the largest number $r$ such that, for any closed halfspace $H \ni x$, there exists a point $y \in \overline{B(x, \varepsilon)}$ such that $B(y, r) \subset H \backslash M$. If $\tau(x, M)=\lim \sup _{\varepsilon \rightarrow 0} \delta_{\varepsilon}(x) / \varepsilon$ then

$$
\tau_{M}=\inf _{x \in M} \tau(x, M)
$$

is called the total porosity of $M$, and $M$ is said to be totally porous if $\tau_{M}>0$. The total porosity was introduced (in metric spaces) by Agronsky and Bruckner [1].

An excellent survey on porosity is Zajicek's paper [13]. About its applications to Convexity see [14]. 
Theorem B. Most functions in $\mathscr{C}(K)$ admit a set of fixed points which is strongly and totally porous.

Proof. Let $f \in \mathscr{C}(K), 0<\alpha<1$ and $0<\beta<\tau_{\text {bd } K}\left(4+\tau_{\text {bd } K}^{2}\right)^{-1 / 2}$. If

$\left(^{*}\right) \forall x \in \mathbb{R}^{d}, \forall \varepsilon>0, \exists y \in \overline{B(x, \varepsilon)}$ s.t. $\overline{B(y, \alpha\|x-y\|)} \cap F_{f}=\varnothing$

then the porosity of $F_{f}$ is at least $\alpha$. If

$$
\left(^{* *}\right) \forall x \in \mathbb{R}^{d}, \forall \varepsilon>0, \forall H \ni x, \exists y \in \overline{B(x, \varepsilon)} \text { s.t. } \overline{B(y, \beta\|x-y\|)} \subset H \backslash F_{f}
$$

then the total porosity of $F_{f}$ is at least $\beta$.

We prove that $\left(^{*}\right)$ and $\left({ }^{* *}\right)$ hold for most $f \in \mathscr{C}(K)$. Let

$$
\mathscr{F}_{n}=\left\{f: \exists x \text { s.t. } \forall y \in \overline{B\left(x, n^{-1}\right)}, \overline{B(y, \alpha\|x-y\|)} \cap F_{f} \neq \emptyset\right\}
$$

and

$$
\mathscr{G}_{n}=\left\{f: \exists x, \exists H \ni x \text { s.t. } \forall y \in \overline{B\left(x, n^{-1}\right)}, \overline{B(y, \beta\|x-y\|)} \not H \backslash F_{f}\right\} .
$$

We now show that the complements of $\mathscr{F}_{n}$ and $\mathscr{G}_{n}$ are dense.

Let $\mathscr{O} \subset \mathscr{C}(K)$ be open. Take $f \in \mathcal{O}$ and $\varepsilon>0$ such that $\|f-g\|<\varepsilon$ implies $g \in \mathcal{O}$. Let $v \in(0, \varepsilon)$ be such that $x, x^{\prime} \in K$ and $\left\|x-x^{\prime}\right\|<v$ imply $\left\|f(x)-f\left(x^{\prime}\right)\right\|<\varepsilon$. From the covering $\left\{B(y, v): y \in F_{f}\right\}$ of $F_{f}$ select a finite subcovering $\{B(y, v): y \in Y\}$. Let $\xi \in\left(0, D\left(K \backslash L, F_{f}\right)\right)$, where

$$
L=\left\{x \in \mathbb{R}^{d}: \Delta(x, Y)<v\right\}
$$

and set

$$
L^{*}=\{x \in L: \Delta(x, \text { bd } L)<\xi\} .
$$

For $\xi$ small enough, the projection (nearest point mapping) $p_{\mathrm{bd} L}: L^{*} \rightarrow$ bd $L$ is single-valued and we assume $\xi$ to be so small. Then $\operatorname{bd}\left(L \backslash L^{*}\right)$ is a differentiable surface and $p_{\mathrm{bd} L}$ is continuous. Let $v$ be a unit vector and construct

$$
f^{*}(x)=\left\{\begin{array}{lc}
f(x) & \text { if } x \in K \backslash L \\
x+\left(1-\xi^{-1}\left\|x-p_{\mathrm{bd} L}(x)\right\|\right) & \left(f\left(p_{K}\left(p_{\mathrm{bd} L}(x)\right)\right)-p_{\mathrm{bd} L}(x)\right) \\
& \text { if } x \in K \cap L^{*} \\
x+\min \left\{\varepsilon, \Delta\left(x, L^{*} \cup \text { bd } K\right)\right\} v & \text { if } x \in K \cap L \backslash L^{*} .
\end{array}\right.
$$

It is easily checked that $f^{*}$ is continuous, that $\left\|f-f^{*}\right\|<\varepsilon$ and that the fixed points of $f^{*}$ form the set $\operatorname{bd}\left(K \cap L \backslash L^{*}\right)$, which is obviously strongly porous. To verify the total porosity of $\operatorname{bd}\left(K \cap L \backslash L^{*}\right)$ we notice:

At every point $y \in\left(\operatorname{bd}\left(K \cap L \backslash L^{*}\right)\right) \backslash\left(\operatorname{bd}\left(L \backslash L^{*}\right)\right), \tau\left(y, F_{f^{*}}\right) \geqq \tau_{\mathrm{bd} K}$. At every point $y^{\prime} \in\left(\mathrm{bd}\left(K \cap L \backslash L^{*}\right)\right) \backslash(\mathrm{bd} K), \tau\left(y^{\prime}, F_{f^{*}}\right)=2^{-1 / 2}$ (the total porosity of a hyperplane). At every point $y^{\prime \prime} \in(\mathrm{bd} K) \cap\left(\operatorname{bd}\left(L \backslash L^{*}\right)\right)$,

$$
\tau\left(y^{\prime \prime}, F_{f^{*}}\right) \geqq \tau_{\text {bd } K}\left(4+\tau_{\text {bd } K}^{2}\right)^{-1 / 2},
$$

because bd $\left(L \backslash L^{*}\right)$ behaves locally like a hyperplane, and the addition of a hyperplane to a set $M$ decreases its total porosity to $\tau_{M}\left(4+\tau_{M}^{2}\right)^{-1 / 2}$, at worst. Therefore

$$
\tau_{F_{f}^{*}} \geqq \tau_{\mathrm{bd} K}\left(4+\tau_{\mathrm{bd} K}^{2}\right)^{-1 / 2}>\beta .
$$

Hence $f^{*} \in \mathcal{O} \backslash\left(\mathscr{F}_{n} \cup \mathscr{G}_{n}\right)$. This shows that the complements of $\mathscr{F}_{n}$ and $\mathscr{G}_{n}$ are dense in $\mathscr{C}(K)$. 
It is easily seen that both $\mathscr{F}_{n}$ and $\mathscr{G}_{n}$ are closed and therefore nowhere dense. Hence $\left({ }^{*}\right)$ and $\left({ }^{* *}\right)$ hold for most $f \in \mathscr{C}(K)$. It follows that, for most functions $f, \rho_{F_{f}} \geqq \alpha$ for any rational $\alpha \in(0,1)$, and $\tau_{F_{f}} \geqq \beta$, which means that $F_{f}$ is strongly and totally porous.

Theorem B and Lebesgue's density theorem imply the following.

Corollary. For most functions in $\mathscr{C}(K)$, the set of fixed points has (d-dimensional) measure zero.

This corollary suggests that the Hausdorff dimension of $F_{f}$ might be lower than $d$. That it is indeed much lower is confirmed by the following result, in which $K$ lives again in a Banach space $E$. I am indebted to David Preiss, who thought of considering the Hausdorff dimension.

Theorem C. Most functions in $\mathscr{C}(K)$ admit a set of fixed points which has Hausdorff dimension 0.

Proof. Let $M \subset E$ and $\alpha>0$. If, for any $\varepsilon>0$, there is a covering $\left\{M_{i}\right\}_{i=1}^{m}$ of $M$ with $\operatorname{diam} M_{i}<\varepsilon$ and $\sum_{i=1}^{m}\left(\operatorname{diam} M_{i}\right)^{\alpha}<\varepsilon$, then the Hausdorff dimension of $M$ is at most $\alpha$. So, let

$$
\begin{gathered}
\mathscr{F}_{n}=\left\{f \in \mathscr{C}(K): \text { if } F_{f} \subset \bigcup_{i=1}^{m} M_{i} \text { for some } m \text { then } \operatorname{diam} \mathrm{M}_{i} \geqq 1 / n\right. \\
\text { for some } \left.i \text { or } \sum_{i=1}^{m}\left(\operatorname{diam} M_{i}\right)^{\alpha} \geqq 1 / n\right\} .
\end{gathered}
$$

We prove that $\mathscr{F}_{n}$ is nowhere dense in $\mathscr{C}(K)$. Indeed, let $\mathcal{O} \subset \mathscr{C}(K)$ be open. By the lemma in the preceding section, we may choose a function $g \in \mathcal{O}$ with $F_{g}$ finite, say $F_{g}=\left\{x_{1}, \ldots, x_{m}\right\}$. Obviously, for any $\beta \in\left(0, \min \left\{(m n)^{-1 / \alpha}, n^{-1}\right\}\right)$, we have $\beta<$ $1 / n$ and $m \beta^{\alpha}<1 / n$, whence $h \notin \mathscr{F}_{n}$ if $F_{h} \subset \bigcup_{i=1}^{m} B\left(x_{i}, \beta / 2\right)$ and this happens in a whole neighbourhood of $g$.

Hence most $f \in \mathscr{C}(K)$ do not belong to $\bigcup_{n=1}^{\infty} \mathscr{F}_{n}$ and have therefore Hausdorff dimension at most $\alpha$. Since this is true for every rational $\alpha>0$, for most $f \in \mathscr{C}(K)$ the Hausdorff dimension of $F_{f}$ is 0 .

\section{References}

1. Agronsky, S., Bruckner, A.: Local compactness and porosity in metric spaces. Real Anal. Exch. 11, 365-379 (1985/6)

2. Baillon, J., Rallis, N.: Not too many fixed points. In: Fixed point theory and its applications. Brown, R. F. (ed.) (Contemp. Math., vol. 72, pp. 21-25) Providence, RI: Am. Math. Soc. 1988

3. Brouwer, L.: Über Abbildung von Mannigfaltigkeiten. Math. Ann. 71, 97-115 (1910)

4. Butler, G.: Almost all 1-set contractions have a fixed point. Proc. Am. Math. Soc. 74, 353-357 (1979)

5. De Blasi, F.: Some generic properties in fixed point theory. J. Math. Anal. Appl. 71, 161-166 (1979)

6. De Blasi, F.: Generic properties for some classes of operator equations. J. Lond. Math. Soc. 23, 321-328 (1981)

7. De Blasi, F., Myjak, J.: Sur la porosité de l'ensemble des contractions sans point fixe. C. R. Acad. Sci., Paris 308, 51-52 (1989)

8. Dominguez Benavides, T.: Some generic properties of $\alpha$-nonexpansive mappings. J. Math. Anal. Appl. 105, 176-187 (1985)

9. Myjak, J.: Orlicz type category theorems for functional and differential equations. Diss. Math. 206, 1-82 (1983)

10. Myjak, J., Sampalmieri, R.: On the porosity of the set of $\omega$-nonexpansive mappings without fixed points (to appear)

11. Schauder, J.: Der Fixpunktsatz in Funktionalräumen. Studia Math. 2, 171-180 (1930) 
12. Vidossich, G.: Existence, uniqueness and approximations of fixed points as a generic property. Bol. Soc. Bras. Math. 5, 17-29 (1974)

13. Zajícek, L.: Porosity and $\sigma$-porosity. Real Anal. Exch. 13, 314-350 (1987/8)

14. Zamfirescu, T.: Porosity in Convexity. Real Anal. Exch. 15, 424-436 (1989/90)

15. Zamfirescu, T:: Baire categories in Convexity. Atti Semin. Mat. Fis. Univ. Modena 39, $139-164(1991)$ 